\title{
Activating remote entanglement in a quantum network by local counting of identical particles
}

\author{
Alessia Castellini, ${ }^{1}$ Bruno Bellomo, ${ }^{2}$ Giuseppe Compagno, ${ }^{1}$ and Rosario Lo Franco ${ }^{1,3, *}$ \\ ${ }^{1}$ Dipartimento di Fisica e Chimica-Emilio Segrè, Università di Palermo, via Archirafi 36, 90123 Palermo, Italy \\ ${ }^{2}$ Institut UTINAM-UMR 6213, CNRS, Université Bourgogne Franche-Comté, \\ Observatoire des Sciences de l'Univers THETA, 41 bis avenue de l'Observatoire, F-25010 Besançon, France \\ ${ }^{3}$ Dipartimento di Ingegneria, Università di Palermo, Viale delle Scienze, Edificio 6, 90128 Palermo, Italy
}

(Received 6 December 2018; published 18 June 2019)

\begin{abstract}
Quantum information and communication processing within quantum networks usually employ identical particles. Despite this, the physical role of the quantum statistical nature of particles in large-scale networks remains elusive. Here, we show that just the indistinguishability of fermions allows a new mechanism of entanglement transfer in many-node quantum networks. This process activates remote entanglement among distant sites, which do not share a common past, by only locally counting identical particles and classical communication. These results constitute the key achievement of the present technique and open the way to a more stable multistage transfer of nonlocal quantum correlations based on fermions.
\end{abstract}

DOI: 10.1103/PhysRevA.99.062322

\section{INTRODUCTION}

New avenues have recently been opened in quantum information and communication by the transfer of quantum states among different separated sites [1]. In fact, it allows distributed quantum computing, a quantum internet, and tests of quantum-mechanics foundations within composite quantum networks [2-4]. Usual state transfer procedures employ identical particles (that are elementary subsystems such as atoms, photons, electrons, qubits), where their entanglement plays an essential role and no effect associated to the statistical nature of the particles typically shows up. This is due to the fact that, in these processes, spatial overlap of the wave functions does not occur in the relevant places so that the identical particles are distinguishable and behave like nonidentical ones. One may then inquire whether employing identical particles may lead to new features in the context of quantum communication by exploiting indistinguishability as a direct resource. For this to happen, it is required to investigate those situations where particles spatially overlap, so that particle identity implies their indistinguishability. Identity of particles has been shown to be useful for some quantum information protocols [5-14] and for quantum metrology $[15,16]$. In this context, one of the problems which remains to investigate is the role played by the quantum statistical nature of identical particles in the mechanisms of entanglement transfer within large-scale networks.

Among the various mechanisms of many-node state transfer, we focus on entanglement swapping (ES), which is a must for large-scale distribution of quantum information [17-20] and is a subject of intense experimental interest [21-26]. ES is an intrinsically quantum phenomenon which permits one to entangle two particles not sharing a common past, each particle being outside the light cone of the other. ES constitutes

\footnotetext{
*rosario.lofranco@unipa.it
}

a key process to implement quantum communication [17-20] and is crucial to build quantum relays and quantum repeaters $[1,27]$. So far, in all the implementations, essential ingredients are the initial creation of entangled pairs and Bell measurements [28]. In the standard ES process [29], two entangled particle pairs are initially prepared and a Bell measurement is successively performed on two particles of different pairs. As a result, the other two particles become entangled even if they never interacted [30,31]. ES has been experimentally realized using identical but distinguishable particles (photons) by applying the usual operational framework for nonidentical particles, based on particle addressability (local operations and classical communication) [32]. The initial entangled pairs of photons are typically created by spontaneous parametric down conversion (SPDC) [21-25]. Recently, ES has been successfully achieved in a quantum network, entangling two photons over a distance of $100 \mathrm{~km}$ [26]. Multiple ESs have been theoretically proposed and experimentally realized by extension of the standard protocol [33-36]. The overall success of the process is influenced by the low creation rate of photon pairs in SPDC [37-39] and by the inefficiency in the realization of Bell measurements [23,28,40-45].

In this paper we present a process of entanglement transfer in a many-node quantum network, where neither initial entangled particle pairs nor Bell measurements are needed, exploiting indistinguishability of fermions. Although the idea of using indistinguishability of identical particles to generate entanglement is not a new one, the present process shows that remote entanglement among distant sites can be generated by only locally counting particles. These characteristics constitute the key achievement of the present technique. In the presence of spatial overlap, the identity of particles makes them individually unaddressable. Therefore, we employ an approach based on spatially localized operations and classical communication (sLOCC), where single-particle local measurements are made onto assigned spatial regions $[13,14]$ (closer to the spirit of quantum field theory). We finally 
compare this process to that with bosons and to a process involving separated intermediate sites.

\section{BASIC PROCESS WITH FERMIONS}

Experimental techniques to control fermions in quantum networks have been recently developed [46-52]. In the following we focus on unveiling remarkable aspects introduced by both fermionic statistics and spatial overlap in the process of remote entanglement distribution.

To this aim, we first describe the basic process which serves as the elementary step for the extension to a many-node quantum network. As displayed in Fig. 1, we take four identical fermions (two pairs), prepared by four independent (spacelike separated) sources $\left\{S_{i}, i=1, \ldots, 4\right\}$. Each particle is sent to the corresponding beam splitter $\mathrm{BS}_{i}$. The two sources $\mathrm{S}_{1}$ and $S_{2}$ independently prepare two fermions with opposite pseudospin. Each beam splitter sends the particle with the same amplitude into two separated sites A and M, so that each particle is in the same delocalized spatial mode $|\alpha\rangle=(|\mathrm{A}\rangle+$ $|M\rangle) / \sqrt{2}$. Similarly, sources $S_{3}$ and $S_{4}$ generate the fermions of the second pair with opposite pseudospin in the delocalized spatial mode $|\beta\rangle=(|\mathrm{M}\rangle+|\mathrm{B}\rangle) / \sqrt{2}$ (right side of Fig. 1). The modes $|\alpha\rangle$ and $|\beta\rangle$ partially overlap in the shared intermediate node $\mathrm{M}$ and the Ith node $(I=\mathrm{A}, \mathrm{M}, \mathrm{B})$ is chosen such that only the localized bound state $|\mathrm{I}\rangle$ is present. The initially prepared four-fermion state $\left|\Psi_{\mathrm{f}}^{(4)}\right\rangle$ can be then formally obtained from $|\alpha \downarrow, \alpha \uparrow, \beta \downarrow, \beta \uparrow\rangle$ by dropping, because of the Pauli exclusion principle, the terms with the same pseudospins in the central node (same spatial state $|\mathbf{M}\rangle$ ) [53]. The ultimate scope is to generate entanglement between particles in the far nodes A and B. This can be achieved by using sLOCC [14], which here consists in a postselection locally counting only one particle of the first pair in A and one particle of the second pair in $\mathrm{B}$ and using classical communication among these sites (this counting implies that in the central node $\mathrm{M}$ there are two particles). The classical communication allows sites A and B to know when an entangled pair is obtained. Notice that the local counting operation is a free operation with respect to

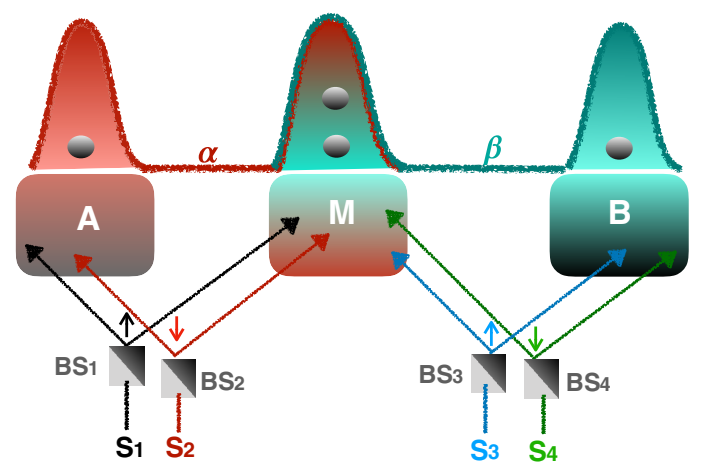

FIG. 1. Basic process. Scheme for the entanglement transfer by four independently prepared indistinguishable fermions, with a shared intermediate node $\mathrm{M}$. The delocalized spatial modes $\alpha$ and $\beta$ partially overlap in correspondence of the intermediate node M. Postselection by sLOCC leaves two fermions with opposite pseudospins in the central node $M$ and two entangled fermions in the extreme nodes, $\mathrm{A}$ and $\mathrm{B}$. entanglement [54]. Such a postselection can be implemented utilizing, for instance, absorptionless particle-counting detectors in A and B, which do not disturb the pseudospin state [9,55-57]. Similar nondemolition measurements are applied in the other postselections by sLOCC used throughout the paper.

One then gets the postselected global state (see Appendix A)

$$
\left|\Psi_{\mathrm{f}, \mathrm{PS}}^{(4)}\right\rangle=\left|\Psi_{\mathrm{M}}, \Psi_{\mathrm{AB}}^{-}\right\rangle
$$

where

$$
\left|\Psi_{\mathrm{M}}\right\rangle=|\mathrm{M} \uparrow, \mathrm{M} \downarrow\rangle,\left|\Psi_{\mathrm{AB}}^{-}\right\rangle=\frac{|\mathrm{A} \downarrow, \mathrm{B} \uparrow\rangle-|\mathrm{A} \uparrow, \mathrm{B} \downarrow\rangle}{\sqrt{2}} .
$$

We have thus obtained a maximally entangled state of twoparticle pseudospins over the distant nodes $\mathrm{A}$ and $\mathrm{B}$, despite the fact that the latter are always independent and the particles do not share any common past. We stress that if the four particles are not identical the same postselection procedure does not give rise to an entangled state. The state of Eq. (1) is obtained with probability (see Appendices A and B) $P_{\mathrm{f}}(4)=\left|\left\langle\Psi_{\mathrm{f}, \mathrm{PS}}^{(4)} \mid \Psi_{\mathrm{f}}^{(4)}\right\rangle\right|^{2}=\left\langle\Psi_{\mathrm{f}}^{(4)}\left|\hat{\Pi}_{\mathrm{f}}\right| \Psi_{\mathrm{f}}^{(4)}\right\rangle=$ $2 / 9$, where $\hat{\Pi}_{\mathrm{f}}=\sum_{\sigma, \tau=\uparrow, \downarrow}|\mathrm{A} \sigma, \mathrm{M} \uparrow, \mathrm{M} \downarrow, \mathrm{B} \tau\rangle\langle\mathrm{A} \sigma, \mathrm{M} \uparrow$, $\mathrm{M} \downarrow, \mathrm{B} \tau \mid$ is the projector onto the (AMB)-operational subspace. We remark that this entanglement distribution is reached without entanglement-inducing Bell measurements on the central particles, but just exploiting the indistinguishability of noninteracting fermions in $\mathrm{M}$. In fact, the spatial overlap of fermions in the shared intermediate site M plays the key role of an entanglement-transfer gate. Schemes for entanglement swapping without Bell measurements have been proposed in contexts where interaction is essential (e.g., cavity QED) $[41,42,58,59]$.

\section{LARGE-SCALE PROCESS WITH FERMIONS}

The basic scheme of Fig. 1 can be straightforwardly iterated to create a remote entanglement transfer in a many-node quantum network. This is achieved by means of $n$ identical fermions and $k=N-1$ shared intermediate nodes $\mathrm{M}_{i}(i=$ $1, \ldots, k)$, where $N=n / 2$ is the number of particle pairs. As displayed in Fig. 2, each $j$ th pair $(j=1, \ldots, N)$ has opposite pseudospins and spatial mode $\left|\alpha_{j}\right\rangle$, with $\left|\alpha_{1}\right\rangle=(|\mathrm{A}\rangle+$ $\left.\left|\mathbf{M}_{1}\right\rangle\right) / \sqrt{2},\left|\alpha_{N}\right\rangle=\left(\left|\mathbf{M}_{k}\right\rangle+|\mathrm{B}\rangle\right) / \sqrt{2}$, and $\left|\alpha_{j}\right\rangle=\left(\left|\mathbf{M}_{j-1}\right\rangle+\right.$ $\left.\left|\mathbf{M}_{j}\right\rangle\right) / \sqrt{2}$ for $j=2, \ldots, k$. The aim is to activate entanglement of particles in the remote far nodes A and B of the network. The modes $\left|\alpha_{i}\right\rangle$ and $\left|\alpha_{i+1}\right\rangle$ partially overlap in the shared intermediate node $\mathrm{M}_{i}$ and the Ith node $\left(\mathrm{I}=\mathrm{A}, \mathrm{M}_{i}\right.$, B) is taken, as already mentioned above, such that only the localized bound state $|\mathrm{I}\rangle$ is present. Once again the initial $n$ fermion state $\left|\Psi_{\mathrm{f}}^{(n)}\right\rangle$ can be formally determined starting from the state $\left|\alpha_{1} \downarrow, \alpha_{1} \uparrow, \ldots, \alpha_{N} \downarrow, \alpha_{N} \uparrow\right\rangle$ simply by dropping, because of the Pauli exclusion principle, the terms having the same pseudospins in each intermediate node. After that, by counting one particle in A and one in B (this entails that each node $\mathrm{M}_{\mathrm{i}}$ contains two particles) and allowing for classical 


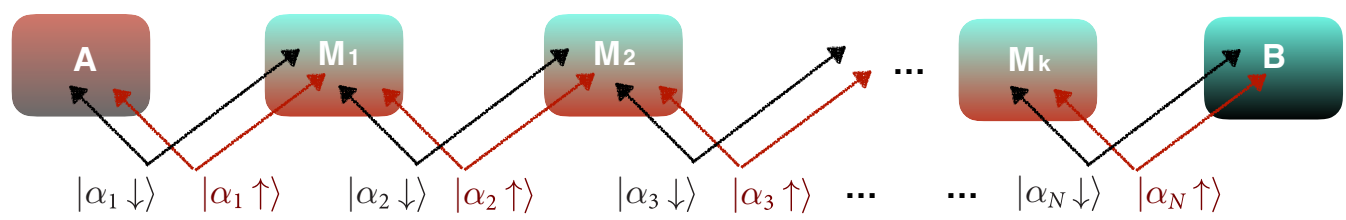

FIG. 2. Many-node quantum network. Scheme for remote entanglement distribution between distant nodes A and B by $n$ independently prepared indistinguishable particles with $k=N-1$ shared intermediate nodes, $N=n / 2$ being the number of involved particle pairs. This process is the straightforward generalization of the scheme of Fig. 1.

communication as before, the postselected global state is

$$
\left|\Psi_{\mathrm{f}, \mathrm{PS}}^{(n)}\right\rangle=\left|\Psi_{\mathrm{M}_{1}}, \Psi_{\mathrm{M}_{2}}, \ldots, \Psi_{\mathrm{M}_{k}}, \Psi_{\mathrm{AB}}^{-}\right\rangle,
$$

where $\left|\Psi_{\mathrm{M}_{i}}\right\rangle=\left|\mathrm{M}_{i} \uparrow, \mathrm{M}_{i} \downarrow\right\rangle$ and $\left|\Psi_{\mathrm{AB}}^{-}\right\rangle$is the maximally entangled (Bell) state of Eq. (2). The probability to obtain the state above is $P_{\mathrm{f}}(n)=\left|\left\langle\Psi_{\mathrm{f}, \mathrm{PS}}^{(n)} \mid \Psi_{\mathrm{f}}^{(n)}\right\rangle\right|^{2}$ (see Appendix A for its explicit expression). Thus, we have generated entanglement of particle pseudospins between the independent distant locations A and B of the many-node network, starting with independently prepared identical fermions, with no Bell measurements and only using local counting operations. We remark that all these features make the remote entanglement activation based on identical fermions deeply different from the standard processes of entanglement transfer such as ES.

\section{PROCESS WITH BOSONS}

The basic setup of Fig. 1 can be also thought to be run by identical bosons. We shall show that, in this case, a Bell measurement onto the intermediate site $\mathrm{M}$ is eventually required for achieving the desired entanglement transfer, similarly to a standard protocol of ES. The sLOCC framework is now realized by locally counting two particles in the intermediate node $\mathrm{M}$ and only one particle in each of the far nodes $\mathrm{A}$ and $\mathrm{B}$, also allowing for classical communication among the different sites. From the initial (un-normalized) state $|\alpha \downarrow, \alpha \uparrow, \beta \downarrow, \beta \uparrow\rangle$, one gets the four-boson postselected state (see Appendix C)

$$
\left|\Psi_{\mathrm{b}, \mathrm{PS}}^{(4)}\right\rangle=\frac{\left|\Psi_{\mathrm{M}}, \Psi_{\mathrm{AB}}^{+}\right\rangle+\left|\Phi_{\mathrm{M}}^{+}, \Phi_{\mathrm{AB}}^{+}\right\rangle-\left|\Phi_{\mathrm{M}}^{-}, \Phi_{\mathrm{AB}}^{-}\right\rangle}{\sqrt{3}},
$$

where $\left|\Phi_{\mathrm{M}}^{ \pm}\right\rangle=(|\mathrm{M} \downarrow, \mathrm{M} \downarrow\rangle \pm|\mathrm{M} \uparrow, \mathrm{M} \uparrow\rangle) / 2,\left|\Psi_{\mathrm{M}}\right\rangle$ is given in Eq. (2), while the distant sites A and B share the Bell states

$$
\begin{aligned}
& \left|\Psi_{\mathrm{AB}}^{+}\right\rangle=\frac{1}{\sqrt{2}}(|\mathrm{~A} \downarrow, \mathrm{B} \uparrow\rangle+|\mathrm{A} \uparrow, \mathrm{B} \downarrow\rangle), \\
& \left|\Phi_{\mathrm{AB}}^{ \pm}\right\rangle=\frac{1}{\sqrt{2}}(|\mathrm{~A} \downarrow, \mathrm{B} \downarrow\rangle \pm|\mathrm{A} \uparrow, \mathrm{B} \uparrow\rangle) .
\end{aligned}
$$

The presence of these three Bell states is a consequence of the fact that bosonic systems admit two-particle states with the same pseudospins in M. As in the standard ES procedure, a joint (Bell) measurement in the shared intermediate node $M$ determines the entangled state in which the first and the last boson of the network collapse, each outcome occurring with probability $p=1 / 3$, as seen from Eq. (4). Since each of the three Bell-state outcomes from the joint measurement in $\mathrm{M}$ realizes the desired entanglement transfer over $\mathrm{A}$ and $\mathrm{B}$, the success probability of the process coincides with the probability of obtaining the postselected state of Eq. (4), which is $P_{\mathrm{b}}(4)=\left|\left\langle\Psi_{\mathrm{b}, \mathrm{PS}}^{(4)} \mid \Psi_{\mathrm{b}}^{(4)}\right\rangle\right|^{2}=6 / 25$ (see Appendices B and $\mathrm{C}$ ). This bosonic protocol can be then extended, analogously to the standard multiple ES, by a cascaded procedure [34]. The scheme remains that of Fig. 2 with $n$ independently prepared identical bosons and $k=N-1$ intermediate nodes $(N=n / 2)$. The sLOCC framework again consists in counting two particles in each intermediate node and one in the distant nodes A and B, also allowing for classical communication of the counting outcomes. One gets the postselected state $\left|\Psi_{\mathrm{b}, \mathrm{PS}}^{(n)}\right\rangle$ and performs Bell measurements step by step on each intermediate node $\mathbf{M}_{i}(i=1, \ldots, k)$ to transfer entanglement over A and B. The success probability is $P_{\mathrm{b}}(n)=\left|\left\langle\Psi_{\mathrm{b}, \mathrm{PS}}^{(n)} \mid \Psi_{\mathrm{b}}^{(n)}\right\rangle\right|^{2}$ (see Appendix C).

\section{PROCESS WITH SEPARATED INTERMEDIATE SITES}

Notice that a procedure much closer to the standard ES can be moreover obtained with indistinguishable particles (bosons or fermions) by employing intermediate separated nodes instead of common intermediate ones.

We take a system made of four identical particles (either bosons or fermions), prepared by four independent (spacelike separated) sources $\left\{S_{i}, i=1, \ldots, 4\right\}$. Each particle is sent to the corresponding beam splitter $\mathrm{BS}_{i}$, as depicted in Fig. 3 . The two sources $S_{1}$ and $S_{2}$ independently prepare two particles with opposite pseudospin. Each beam splitter sends the particle with the same amplitude into two separated sites A and $\mathrm{C}$, so that each particle is in the same delocalized spatial

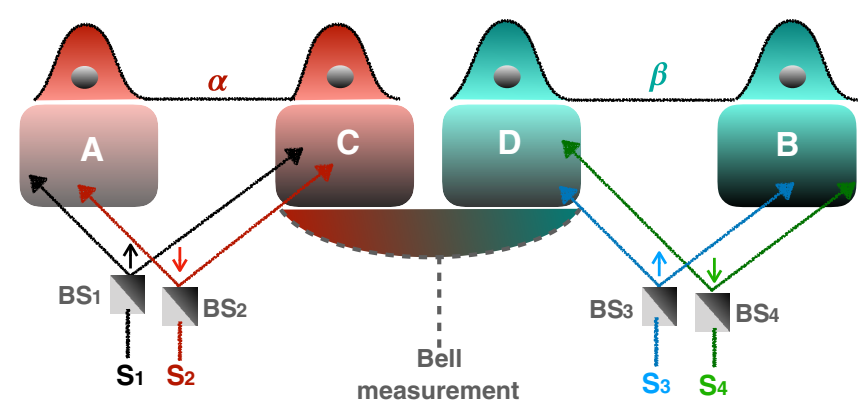

FIG. 3. Four-node scheme for the entanglement swapping by indistinguishable particles (bosons or fermions). Four independent single-particle sources $S_{i}(i=1, \ldots, 4)$ send each particle to the corresponding beam splitter $\left(\mathrm{BS}_{i}\right) . \alpha$ and $\beta$ are the (delocalized) spatial modes peaked in correspondence of the separated spatial nodes A-C and D-B, respectively. Postselection by sLOCC leaves only one particle in each node and Bell measurements are finally performed. 
mode $|\alpha\rangle=(|\mathrm{A}\rangle+|\mathrm{C}\rangle) / \sqrt{2}$. Similarly, sources $\mathrm{S}_{3}$ and $\mathrm{S}_{4}$ generate the particles of the second pair with opposite pseudospin in the delocalized spatial mode $|\beta\rangle=(|\mathrm{D}\rangle+|\mathrm{B}\rangle) / \sqrt{2}$ (right side of Fig. 3). All the nodes are spatially separated (the modes $|\alpha\rangle$ and $|\beta\rangle$ are orthogonal) and the Ith node $(\mathrm{I}=\mathrm{A}, \mathrm{B}, \mathrm{C}, \mathrm{D})$ is chosen such that only the localized bound state $|\mathrm{I}\rangle$ is present. The global four-particle quantum state [10] is therefore $\left|\Psi^{(4)}\right\rangle=|\alpha \downarrow, \alpha \uparrow, \beta \downarrow, \beta \uparrow\rangle$. From this state it is possible to obtain entanglement in the pseudospin degrees of freedom linked to the spatial overlap of particles in each pair. This is achieved by sLOCC consisting in a postselection counting only one particle of the first pair in A and one particle of the second pair in $\mathrm{B}$ and classically communicating the outcomes to each other. As a result, each node contains one particle and we obtain the state (see Appendix D)

$$
\left|\Psi_{\mathrm{PS}}^{(4)}\right\rangle=\left|\Psi_{\mathrm{AC}}, \Psi_{\mathrm{DB}}\right\rangle,
$$

where $\left|\Psi_{\mathrm{AC}}\right\rangle$ and $\left|\Psi_{\mathrm{DB}}\right\rangle$ are the two-particle Bell states

$$
\begin{aligned}
& \left|\Psi_{\mathrm{AC}}\right\rangle=\frac{1}{\sqrt{2}}(|\mathrm{~A} \downarrow, \mathrm{C} \uparrow\rangle+\eta|\mathrm{A} \uparrow, \mathrm{C} \downarrow\rangle), \\
& \left|\Psi_{\mathrm{DB}}\right\rangle=\frac{1}{\sqrt{2}}(|\mathrm{D} \downarrow, \mathrm{B} \uparrow\rangle+\eta|\mathrm{D} \uparrow, \mathrm{B} \downarrow\rangle) .
\end{aligned}
$$

Even if the particles have been independently prepared, as a consequence of sLOCC, the state $\left|\Psi_{\mathrm{PS}}^{(4)}\right\rangle$ shows that the pair of particles in $\mathrm{A}$ and $\mathrm{C}$ is maximally entangled in the pseudospin degrees of freedom, as the DB pair. This state is obtained with probability $P(4)=\left|\left\langle\Psi_{\mathrm{PS}}^{(4)} \mid \Psi^{(4)}\right\rangle\right|^{2}=1 / 4$ (see Appendix D). At this stage the particles can be distinguished, since they are in spatially separated sites. We stress that for each pair, if the particles are not identical, the same postselection procedure does not give rise to an entangled state. The structure of the state of Eq. (6) allows one to implement the standard protocol of ES [29]: performing a Bell measurement on near central nodes $\mathrm{C}$ and $\mathrm{D}$ transfers entanglement to the particles in the far nodes A and B. Notice that this procedure does not require, at the preparation stage, two entangled pairs. The present scheme works for both bosons and fermions, also when particles of different pairs are not identical. Moreover, in analogy with the standard ES, it can be naturally iterated by a cascaded procedure [34] to realize multistage entanglement swapping with $n=2 N$ independently prepared particles, $N$ being the number of involved particle pairs. This is achieved by using a network with $n-2$ separated central nodes, where each pair of identical particles (either bosons or fermions) is prepared with opposite pseudospins in an equal delocalized spatial mode peaked in correspondence of two separated nodes (as shown for the two pairs in Fig. 3). After obtaining a single particle in each central node and performing Bell measurements step by step onto two central nodes [34], one eventually entangles the particles in the extreme nodes of the network with probability $P(n)=1 / 2^{N}$. In Appendix E, the success probabilities of the various protocols above are compared.

\section{CONCLUSIONS}

In this paper we have presented a conceptual process of entanglement distribution in a large-scale quantum network which is fundamentally activated by indistinguishability of particles. The standard entanglement swapping, that is, the renowned process for entanglement transfer with distinguishable particles, necessitates to start from entangled particle pairs and requires final Bell measurements [29]. Compared to this one, the present process, run by identical fermions, enables remote entanglement among distant nodes through the following different aspects: (i) with no distribution of initial entangled pairs and (ii) without performing Bell measurements, because of the natural entanglement due to spatially overlapping identical particles. In fact, the process only requires local counting of independently prepared identical particles. The measurement process, when described on the level of particles, looks different for indistinguishable and distinguishable particles.

The key advantage of this process is that it simplifies the task of distributing entanglement, overcoming the drawbacks encountered in the usual entanglement transfer procedures during the initial preparation stage and the final measurement phase. In fact, it skips the use of sources of entangled particle pairs, which are, for instance, generated by SPDC at the very low rate of about $10^{-2}$ for a single laser pulse [38], and also avoids the experimental inefficiency associated to performing Bell measurements [23,28,40-45].

The proposed fermionic process could be, for instance, realized by using quantum dots as sources of single electrons that can be initialized in particular spin states [47], emitted on demand [48], and directed to quantum point contacts acting as electronic beam splitters $[49,60]$. Single electrons have been also recently shown to be controlled within atomic circuits [50]. Moreover, further setups in quantum optics, simulating fermionic statistics using photons, and integrated photonics [51,52] could, in principle, represent convenient platforms.

Our results reveal once more [14] that spatial overlap of identical particles constitutes an operational resource. In addition, they pave the way to a more stable multistage remote entanglement transfer based on fermions, evidencing the effect of the quantum statistical nature of particles on quantum information processing.

\section{ACKNOWLEDGMENTS}

A.C. acknowledges useful discussions with Dario Cilluffo, Mauro Valeri, Andrea Geraldi, and Emanuele Polino.

\section{APPENDIX A: EXPLICIT CALCULATIONS FOR SHARED INTERMEDIATE SITES WITH FERMIONS}

The four-fermion global state $\left|\Psi_{\mathrm{f}}^{(4)}\right\rangle$ is

$$
\begin{aligned}
\left|\Psi_{\mathrm{f}}^{(4)}\right\rangle= & \frac{1}{3}(|\mathrm{~A} \downarrow, \mathrm{A} \uparrow, \mathrm{M} \downarrow, \mathrm{M} \uparrow\rangle+|\mathrm{A} \downarrow, \mathrm{A} \uparrow, \mathrm{M} \downarrow, \mathrm{B} \uparrow\rangle \\
& +|\mathrm{A} \downarrow, \mathrm{A} \uparrow, \mathrm{B} \downarrow, \mathrm{M} \uparrow\rangle+|\mathrm{A} \downarrow, \mathrm{A} \uparrow, \mathrm{B} \downarrow, \mathrm{B} \uparrow\rangle \\
& +|\mathrm{A} \downarrow, \mathrm{M} \uparrow, \mathrm{M} \downarrow, \mathrm{B} \uparrow\rangle+|\mathrm{A} \downarrow, \mathrm{M} \uparrow, \mathrm{B} \downarrow, \mathrm{B} \uparrow\rangle \\
& +|\mathrm{M} \downarrow, \mathrm{A} \uparrow, \mathrm{B} \downarrow, \mathrm{M} \uparrow\rangle+|\mathrm{M} \downarrow, \mathrm{A} \uparrow, \mathrm{B} \downarrow, \mathrm{B} \uparrow\rangle \\
& +|\mathrm{M} \downarrow, \mathrm{M} \uparrow, \mathrm{B} \downarrow, \mathrm{B} \uparrow\rangle) .
\end{aligned}
$$

The sLOCC here consists in counting one particle in A and one in B (this entails having two particles in $\mathrm{M}$ ) and allowing for classical communication of the outcomes. Projecting, 
therefore, the above prepared state onto the subspace spanned by the basis $\mathcal{B}_{\mathrm{f}}=\{|\mathrm{A} \sigma, \mathrm{M} \uparrow, \mathrm{M} \downarrow, \mathrm{B} \tau\rangle\}(\sigma, \tau=\downarrow, \uparrow)$, we find the postselected state

$$
\left|\Psi_{\mathrm{f}, \mathrm{PS}}^{(4)}\right\rangle=|\mathrm{M} \uparrow, \mathrm{M} \downarrow\rangle \wedge\left|\Psi_{\mathrm{AB}}^{-}\right\rangle,
$$

which is obtained with probability $P_{\mathrm{f}}(4)=\left|\left\langle\Psi_{\mathrm{f}, \mathrm{PS}}^{(4)} \mid \Psi_{\mathrm{f}}^{(4)}\right\rangle\right|^{2}=$ 2/9 (see Appendix B). The state $\left|\Psi_{\mathrm{AB}}^{-}\right\rangle$is the maximally Bell state of Eq. (2). In Eq. (A2) we have used the wedge product $\wedge$ that, in this case of separated sites under sLOCC, coincides with the standard tensor product [13].

The scheme presented for the minimum core with four particles can be extended to the case of $n=2 N$ particles, where $N$ is the number of involved particle pairs. The generalized scheme with $k=N-1$ shared intermediate nodes $\mathrm{M}_{i}$ $(i=1, \ldots, k)$ is displayed in Fig. 1 of the main text.

Each $j$ th pair $(j=1, \ldots, N)$ has opposite pseudospins and (delocalized) spatial mode $\left|\alpha_{j}\right\rangle$, with $\left|\alpha_{1}\right\rangle=(|\mathrm{A}\rangle+$ $\left.\left|\mathrm{M}_{1}\right\rangle\right) / \sqrt{2},\left|\alpha_{N}\right\rangle=\left(\left|\mathrm{M}_{k}\right\rangle+|\mathrm{B}\rangle\right) / \sqrt{2}$, and $\left|\alpha_{j}\right\rangle=\left(\left|\mathrm{M}_{j-1}\right\rangle+\right.$ $\left.\left|\mathrm{M}_{j}\right\rangle\right) / \sqrt{2}$ for $j=2, \ldots, k$. We take as the initially prepared $n$-fermion state $\left|\Psi_{\mathrm{f}}^{(n)}\right\rangle$ the one obtained from $\left|\alpha_{1} \downarrow, \alpha_{1} \uparrow, \ldots, \alpha_{N} \downarrow, \alpha_{N} \uparrow\right\rangle$ by eliminating, because of the Pauli exclusion principle, the terms with two particles in the same node with the same pseudospin. The normalization constant $\mathcal{N}_{\mathrm{f}}$ of $\left|\Psi_{\mathrm{f}}^{(n)}\right\rangle$ can be conveniently expressed as $\mathcal{N}_{\mathrm{f}}=$ $\sqrt{\operatorname{det}\left(\mathcal{M}^{(n)}\right)}$ where $\operatorname{det}\left(\mathcal{M}^{(n)}\right)$ is the determinant of the $n \times n$ matrix

$$
\mathcal{M}^{(n)}=\left(\begin{array}{ccc}
\left\langle\alpha_{1} \downarrow \mid \alpha_{1} \downarrow\right\rangle & \cdots & \left\langle\alpha_{1} \downarrow \mid \alpha_{N} \uparrow\right\rangle \\
\vdots & \ddots & \vdots \\
\left\langle\alpha_{N} \uparrow \mid \alpha_{1} \downarrow\right\rangle & \cdots & \left\langle\alpha_{N} \uparrow \mid \alpha_{N} \uparrow\right\rangle
\end{array}\right),
$$

defined in the $n$-dimensional one-particle basis $\left\{\left|\alpha_{1} \downarrow\right\rangle,\left|\alpha_{1} \uparrow\right\rangle,\left|\alpha_{2} \downarrow\right\rangle,\left|\alpha_{2} \uparrow\right\rangle, \ldots,\left|\alpha_{N} \downarrow\right\rangle,\left|\alpha_{N} \uparrow\right\rangle\right\}$.

The sLOCC framework is once again realized by counting one particle in each of the nodes $\mathrm{A}$ and $\mathrm{B}$ (this entails that two particles are in each node $\mathrm{M}_{i}$ ), also allowing for classical communication of the counting outcomes. The postselected global state results in

$$
\left|\Psi_{\mathrm{f}, \mathrm{PS}}^{(n)}\right\rangle=\left|\Psi_{\mathrm{M}_{1}}, \Psi_{\mathrm{M}_{2}}, \ldots, \Psi_{\mathrm{M}_{k}}, \Psi_{\mathrm{AB}}^{-}\right\rangle,
$$

where $\left|\Psi_{\mathrm{M}_{i}}\right\rangle=\left|\mathrm{M}_{i} \uparrow, \mathrm{M}_{i} \downarrow\right\rangle$ and $\left|\Psi_{\mathrm{AB}}^{-}\right\rangle$is a maximally entangled Bell state [see Eq. (2)]. The probability to obtain the state above, that is, the success probability of the remote entanglement transfer process, is given by $P_{\mathrm{f}}(n)=\left|\left\langle\Psi_{\mathrm{f}, \mathrm{PS}}^{(n)} \mid \Psi_{\mathrm{f}}^{(n)}\right\rangle\right|^{2}$. Using the formalism presented in Appendix B, it is straightforward to show that its explicit expression as a function of the number of fermions is

$$
P_{\mathrm{f}}(n)=\frac{1}{2^{n-1} \operatorname{det}\left(\mathcal{M}^{(n)}\right)} .
$$

\section{APPENDIX B: SCALAR PRODUCTS IN THE NO-LABEL FORMALISM}

For calculating the success probabilities of the proposed protocols under different configurations, we need to compute scalar products between states of $n$ identical particles. These are obtained by the $n$-particle probability amplitude defined in the nonstandard approach (no-label particle-based formalism) here adopted $[10,13]$, the general expression of which is

$$
\begin{aligned}
& \left\langle\varphi_{1}^{\prime}, \varphi_{2}^{\prime}, \ldots, \varphi_{n}^{\prime} \mid \varphi_{1}, \varphi_{2}, \ldots, \varphi_{n}\right\rangle \\
& :=\sum_{P} \eta^{P}\left\langle\varphi_{1}^{\prime} \mid \varphi_{P_{1}}\right\rangle\left\langle\varphi_{2}^{\prime} \mid \varphi_{P_{2}}\right\rangle \ldots\left\langle\varphi_{n}^{\prime} \mid \varphi_{P_{n}}\right\rangle,
\end{aligned}
$$

where $P=\left\{P_{1}, P_{2}, \ldots, P_{n}\right\}$ in the sum runs over all the oneparticle state permutations, $\eta= \pm 1$ for bosons and fermions, respectively, and $\eta^{P}$ is 1 for bosons and $1(-1)$ for even (odd) permutations for fermions.

\section{APPENDIX C: EXPLICIT CALCULATIONS FOR SHARED INTERMEDIATE SITES WITH BOSONS}

The four-boson global state $\left|\Psi_{\mathrm{b}}^{(4)}\right\rangle$ is

$\left|\Psi_{\mathrm{b}}^{(4)}\right\rangle=\frac{1}{5}|(\mathrm{~A}+\mathrm{M}) \downarrow,(\mathrm{A}+\mathrm{M}) \uparrow,(\mathrm{M}+\mathrm{B}) \downarrow,(\mathrm{M}+\mathrm{B}) \uparrow\rangle$.

The basis for sLOCC, corresponding to counting two particles in the shared intermediate mode $\mathrm{M}$ and one particle in each mode $\mathrm{A}$ and $\mathrm{B}$, is $\mathcal{B}_{\mathrm{b}}=\left\{\frac{\left|\mathrm{A} \sigma, \mathrm{M} \tau, \mathrm{M} \sigma^{\prime}, \mathrm{B} \tau^{\prime}\right\rangle}{\mathcal{N}_{\tau \sigma^{\prime}}}\right\}\left(\sigma, \tau, \sigma^{\prime}, \tau^{\prime}=\downarrow\right.$ , $\uparrow)$ where $\mathcal{N}_{\tau \sigma^{\prime}}=\sqrt{1+\left\langle\tau \mid \sigma^{\prime}\right\rangle}$. The four-boson postselected state, after projection onto $\mathcal{B}_{\mathrm{b}}$ (also allowing for classical communication), is then

$$
\begin{aligned}
\left|\Psi_{\mathrm{b}, \mathrm{PS}}^{(4)}\right\rangle= & \frac{1}{\sqrt{6}}(|\mathrm{~A} \downarrow, \mathrm{M} \uparrow, \mathrm{M} \uparrow, \mathrm{B} \downarrow\rangle+|\mathrm{A} \downarrow, \mathrm{M} \uparrow, \mathrm{M} \downarrow, \mathrm{B} \uparrow\rangle \\
& +|\mathrm{A} \uparrow, \mathrm{M} \uparrow, \mathrm{M} \downarrow, \mathrm{B} \downarrow\rangle+|\mathrm{A} \uparrow, \mathrm{M} \downarrow, \mathrm{M} \downarrow, \mathrm{B} \uparrow\rangle),
\end{aligned}
$$

which is found with probability $P_{\mathrm{b}}(4)=\left|\left\langle\Psi_{\mathrm{b}, \mathrm{PS}}^{(4)} \mid \Psi_{\mathrm{b}}^{(4)}\right\rangle\right|^{2}=$ $6 / 25$.

In the M subspace, the Bell basis for bosons is given by the three states $\left|\Phi_{\mathrm{M}}^{ \pm}\right\rangle=(|\mathrm{M} \downarrow, \mathrm{M} \downarrow\rangle \pm|\mathrm{M} \uparrow, \mathrm{M} \uparrow\rangle) / 2$ and $\left|\Psi_{\mathrm{M}}\right\rangle=|\mathrm{M} \uparrow, \mathrm{M} \downarrow\rangle$. The postselected state $\left|\Psi_{\mathrm{b}, \mathrm{PS}}^{(4)}\right\rangle$ can be then expressed in terms of this Bell basis as

$$
\left|\Psi_{\mathrm{b}, \mathrm{PS}}^{(4)}\right\rangle=\frac{\left|\Psi_{\mathrm{M}}, \Psi_{\mathrm{AB}}^{+}\right\rangle+\left|\Phi_{\mathrm{M}}^{+}, \Phi_{\mathrm{AB}}^{+}\right\rangle-\left|\Phi_{\mathrm{M}}^{-}, \Phi_{\mathrm{AB}}^{-}\right\rangle}{\sqrt{3}},
$$

where in each term the particles in the distant sites A and B are in a Bell state [see Eq. (5)]. Therefore, each outcome of the joint Bell measurement successfully realizes the entanglement swapping over the distant nodes A and B.

The four-particle bosonic protocol can be extended, analogously to the standard ES, by a cascaded procedure [34]. The scheme is again that of Fig. 2 with $n$ independently prepared identical bosons and $k=N-1$ intermediate nodes $(N=n / 2$ is the number of particle pairs).

The initially prepared $n$-boson state is

$$
\left|\Psi_{\mathrm{b}}^{(n)}\right\rangle=\frac{1}{\mathcal{N}_{b}}\left|\alpha_{1} \downarrow, \alpha_{1} \uparrow, \alpha_{2} \downarrow, \alpha_{2} \uparrow, \ldots, \alpha_{N} \downarrow, \alpha_{N} \uparrow\right\rangle,
$$

where the normalization constant $\mathcal{N}_{b}=\sqrt{\operatorname{perm}\left(\mathcal{M}^{(n)}\right)}$, with $\operatorname{perm}\left(\mathcal{M}^{(n)}\right)$ being the permanent of the matrix of Eq. (A3).

By counting two particles in each intermediate node and one in each of the distant nodes $\mathrm{A}$ and $\mathrm{B}$, also allowing for classical communication of the counting outcomes, one gets the postselected state $\left|\Psi_{\mathrm{b}, \mathrm{PS}}^{(n)}\right\rangle$. Bell measurements are then performed step by step on each intermediate node $\mathbf{M}_{i}(i=$ $1, \ldots, k)$ to transfer entanglement over A and B. The type of 
the final Bell state transferred over A and B will depend on the consecutive outcomes of the cascaded Bell measurements. The success probability of the protocol is obtained by $P_{\mathrm{b}}(n)=$ $\left|\left\langle\Psi_{\mathrm{b}, \mathrm{PS}}^{(n)} \mid \Psi_{\mathrm{b}}^{(n)}\right\rangle\right|^{2}$. Its explicit expression as a function of the number of bosons, in the cases of Fig. 4, is

$$
P_{\mathrm{b}}(n)=\frac{3^{\frac{n}{2}-1}}{2^{n-1} \operatorname{perm}\left(\mathcal{M}^{(n)}\right)} .
$$

In order to be more explicit concerning the cascaded procedure leading to the Bell states over A and B, we treat the case with $n=6$ bosons $(N=3$ pairs $)$ and two shared intermediate nodes $\mathrm{M}_{1}$ and $\mathrm{M}_{2}$. From the initially prepared state $\left|\Psi_{\mathrm{b}}^{(6)}\right\rangle$, easily obtained from Eq. (C4), the sLOCC framework counting two particles in the intermediate nodes and one particle in each of the far nodes $\mathrm{A}$ and $\mathrm{B}$, including classical communication, leads to the postselected state

$$
\begin{aligned}
\left|\Psi_{\mathrm{b}, \mathrm{PS}}^{(6)}\right\rangle= & \frac{\sqrt{2}}{3}\left(\left|\Psi_{\mathrm{M}_{1}}, \Psi_{\mathrm{AM}_{2}}^{+}\right\rangle+\left|\Phi_{\mathrm{M}_{1}}^{+}, \Phi_{\mathrm{AM}_{2}}^{+}\right\rangle\right. \\
& \left.-\left|\Phi_{\mathrm{M}_{1}}^{-}, \Phi_{\mathrm{AM}_{2}}^{-}\right\rangle\right) \wedge\left|\Psi_{\mathrm{M}_{2} \mathrm{~B}}^{+}\right\rangle,
\end{aligned}
$$

where the relevant Bell states are analogous to those given after Eq. (C2) and in Eq. (5). A first Bell measurement has to be performed on the intermediate node $M_{1}$ in order to entangle bosons in $\mathrm{A}$ and $\mathrm{M}_{2}$. Any outcome is good for continuing the protocol. Let us suppose, without loss of generality, that the result of this first Bell measurement is $\left|\Phi_{\mathrm{M}_{1}}^{-}\right\rangle$. From $\left|\Psi_{\mathrm{b}, \mathrm{PS}}^{(6)}\right\rangle$ above, one sees that the remaining four bosons are left in the state $-\left|\Phi_{\mathrm{AM}_{2}}^{-}, \Psi_{\mathrm{M}_{2} \mathrm{~B}}^{+}\right\rangle$. This state, suitably normalized and expressed in terms of the Bell basis in the node $\mathrm{M}_{2}$, assumes the form

$$
\left|\Psi^{(4)}\right\rangle_{\mathrm{AM}_{2} \mathrm{~B}}=\frac{\left|\Phi_{\mathrm{M}_{2}}^{+}, \Psi_{\mathrm{AB}}^{-}\right\rangle+\left|\Phi_{\mathrm{M}_{2}}^{-}, \Psi_{\mathrm{AB}}^{+}\right\rangle+\left|\Psi_{\mathrm{M}_{2}}, \Phi_{\mathrm{AB}}^{-}\right\rangle}{\sqrt{3}} .
$$

It is now clear that a second Bell measurement on the intermediate node $\mathrm{M}_{2}$ has the final effect to transfer a Bell state over the far nodes $\mathrm{A}$ and $\mathrm{B}$. Thus, for six bosons, two cascaded Bell measurements realize the desired entanglement swapping protocol. This procedure can be continued analogously for successive steps with more particles.

\section{APPENDIX D: EXPLICIT CALCULATIONS FOR SEPARATED INTERMEDIATE SITES}

Here, we report the calculations regarding the scheme depicted in Fig. 3. The prepared state $\left|\Psi^{(4)}\right\rangle$ can be written as the superposition of 16 terms:

$$
\begin{aligned}
\left|\Psi^{(4)}\right\rangle= & \frac{1}{4}(|\mathrm{~A} \downarrow, \mathrm{A} \uparrow, \mathrm{D} \downarrow, \mathrm{D} \uparrow\rangle+|\mathrm{A} \downarrow, \mathrm{A} \uparrow, \mathrm{D} \downarrow, \mathrm{B} \uparrow\rangle \\
& +|\mathrm{A} \downarrow, \mathrm{A} \uparrow, \mathrm{B} \downarrow, \mathrm{D} \uparrow\rangle+|\mathrm{A} \downarrow, \mathrm{A} \uparrow, \mathrm{B} \downarrow, \mathrm{B} \uparrow\rangle \\
& +|\mathrm{A} \downarrow, \mathrm{C} \uparrow, \mathrm{D} \downarrow, \mathrm{D} \uparrow\rangle+|\mathrm{A} \downarrow, \mathrm{C} \uparrow, \mathrm{D} \downarrow, \mathrm{B} \uparrow\rangle \\
& +|\mathrm{A} \downarrow, \mathrm{C} \uparrow, \mathrm{B} \downarrow, \mathrm{D} \uparrow\rangle+|\mathrm{A} \downarrow, \mathrm{C} \uparrow, \mathrm{B} \downarrow, \mathrm{B} \uparrow\rangle \\
& +|\mathrm{C} \downarrow, \mathrm{A} \uparrow, \mathrm{D} \downarrow, \mathrm{D} \uparrow\rangle+|\mathrm{C} \downarrow, \mathrm{A} \uparrow, \mathrm{D} \downarrow, \mathrm{B} \uparrow\rangle \\
& +|\mathrm{C} \downarrow, \mathrm{A} \uparrow, \mathrm{B} \downarrow, \mathrm{D} \uparrow\rangle+|\mathrm{C} \downarrow, \mathrm{A} \uparrow, \mathrm{B} \downarrow, \mathrm{B} \uparrow\rangle \\
& +|\mathrm{C} \downarrow, \mathrm{C} \uparrow, \mathrm{D} \downarrow, \mathrm{D} \uparrow\rangle+|\mathrm{C} \downarrow, \mathrm{C} \uparrow, \mathrm{D} \downarrow, \mathrm{B} \uparrow\rangle \\
& +|\mathrm{C} \downarrow, \mathrm{C} \uparrow, \mathrm{B} \downarrow, \mathrm{D} \uparrow\rangle+|\mathrm{C} \downarrow, \mathrm{C} \uparrow, \mathrm{B} \downarrow, \mathrm{B} \uparrow\rangle) .
\end{aligned}
$$

In the linear combination of Eq. (D1), there are contributions in which two particles occupy the same site. We perform sLOCC in the form of a postselection counting a single particle in each site A and B and classically communicating their outcomes to each other (notice that a single particle in A entails one particle in $\mathrm{C}$ and a single particle in $\mathrm{B}$ implies one particle in D). This postselection corresponds to project the global four-particle state $\left|\Psi^{(4)}\right\rangle$ onto the subspace spanned by the spatially localized basis $\mathcal{B}=\left\{\left|\mathrm{A} \sigma, \mathrm{C} \tau, \mathrm{D} \sigma^{\prime}, \mathrm{B} \tau^{\prime}\right\rangle\right\}$, by the projector $\hat{\Pi}_{\mathrm{ACDB}}=$ $\sum_{\sigma, \tau, \sigma^{\prime}, \tau^{\prime}=\downarrow, \uparrow}\left|\mathrm{A} \sigma, \mathrm{C} \tau, \mathrm{D} \sigma^{\prime}, \mathrm{B} \tau^{\prime}\right\rangle\left\langle\mathrm{A} \sigma, \mathrm{C} \tau, \mathrm{D} \sigma^{\prime}, \mathrm{B} \tau^{\prime}\right|$.

The postselected (projected) state is thus obtained as

$$
\left|\Psi_{\mathrm{PS}}^{(4)}\right\rangle=\hat{\Pi}_{\mathrm{ACDB}}\left|\Psi^{(4)}\right\rangle / \mathcal{N},
$$

where $\mathcal{N}=\sqrt{\left\langle\Psi^{(4)}\left|\hat{\Pi}_{\mathrm{ACDB}}\right| \Psi^{(4)}\right\rangle}=1 / 2$. Its explicit expression is

$$
\begin{aligned}
\left|\Psi_{\mathrm{PS}}^{(4)}\right\rangle= & \frac{1}{\sqrt{2}}(|\mathrm{~A} \downarrow, \mathrm{C} \uparrow\rangle+\eta|\mathrm{A} \uparrow, \mathrm{C} \downarrow\rangle) \\
& \wedge \frac{1}{\sqrt{2}}(|\mathrm{D} \downarrow, \mathrm{B} \uparrow\rangle+\eta|\mathrm{D} \uparrow, \mathrm{B} \downarrow\rangle),
\end{aligned}
$$

that is, $\left|\Psi_{\mathrm{PS}}^{(4)}\right\rangle=\left|\Psi_{\mathrm{AC}}, \Psi_{\mathrm{DB}}\right\rangle$ [see Eqs. (6) and (7)]. This state is obtained with probability $P(4)=\left|\left\langle\Psi_{\mathrm{PS}}^{(4)} \mid \Psi^{(4)}\right\rangle\right|^{2}=$ $1 / 4$. Notice that in this sLOCC framework the prepared state $\left|\Psi^{(4)}\right\rangle$ can be written as $\left|\Psi^{(4)}\right\rangle=|\alpha \downarrow, \alpha \uparrow\rangle \wedge|\beta \downarrow, \beta \uparrow\rangle$, from which one then obtains particle entanglement between (A, C) and (D, B), as evidenced in Eq. (D3). This is linked to the concept of indistinguishability as a resource by sLOCC introduced in Ref. [14].

Since the sites (A, C) and (D, B) are separated, the identical particles can be distinguished by their spatial location. Once the postselected state $\left|\Psi_{\mathrm{PS}}^{(4)}\right\rangle$ is obtained, the entanglement swapping proceeds following the lines of the standard protocol for distinguishable particles [29]. A Bell measurement is therefore performed on the intermediate nodes $(C, D)$ to obtain entanglement over the far nodes A and B. In fact, one can write

$$
\begin{aligned}
\left|\Psi_{\mathrm{PS}}^{(4)}\right\rangle= & \frac{1}{2}\left[\left|\Psi_{\mathrm{CD}}^{+}, \Psi_{\mathrm{AB}}^{+}\right\rangle-\left|\Psi_{\mathrm{CD}}^{-}, \Psi_{\mathrm{AB}}^{-}\right\rangle\right. \\
& \left.+\eta\left|\Phi_{\mathrm{CD}}^{+}, \Phi_{\mathrm{AB}}^{+}\right\rangle-\eta\left|\Phi_{\mathrm{CD}}^{-}, \Phi_{\mathrm{AB}}^{-}\right\rangle\right],
\end{aligned}
$$

where

$$
\begin{aligned}
& \left|\Psi_{\mathrm{IJ}}^{ \pm}\right\rangle=\frac{1}{\sqrt{2}}(|\mathrm{I} \downarrow, \mathrm{J} \uparrow\rangle \pm|\mathrm{I} \uparrow, \mathrm{J} \downarrow\rangle), \\
& \left|\Phi_{\mathrm{IJ}}^{ \pm}\right\rangle=\frac{1}{\sqrt{2}}(|\mathrm{I} \downarrow, \mathrm{J} \downarrow\rangle \pm|\mathrm{I} \uparrow, \mathrm{J} \uparrow\rangle),
\end{aligned}
$$

with $\mathrm{IJ}=\mathrm{AB}, \mathrm{CD}$. The result of the Bell measurement does not depend on the particle statistics, as expected from the fact that the postselected state describes identical particles in separated spatial regions under sLOCC.

The previous protocol can be straightforwardly extended to multiple entanglement swapping (with $n=2 N$ independently prepared particles, $N$ being the number of involved particle pairs), in analogy to the case of distinguishable particles [34], 


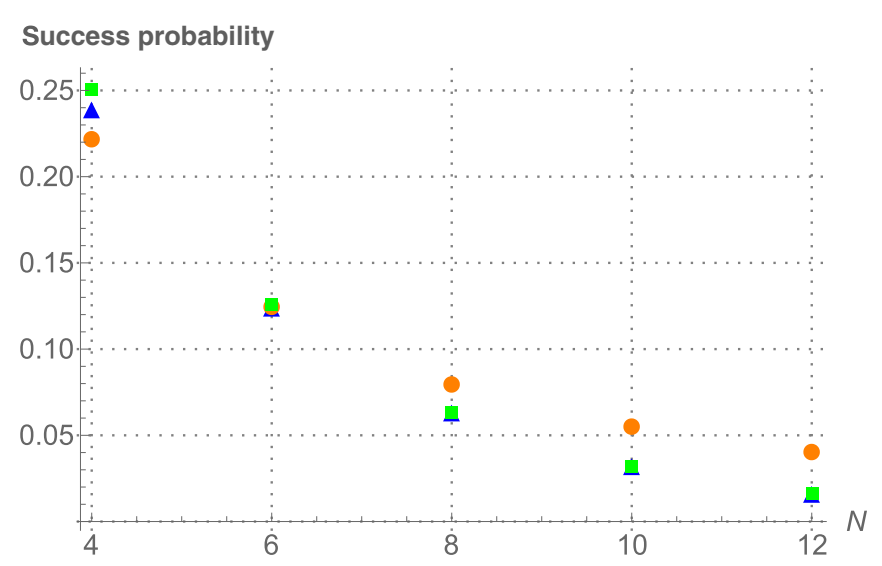

FIG. 4. Success probability to implement multiple entanglement transfer as a function of the number of particles $n$, for separated nodes with either bosons or fermions $[P(n)$, green squares] and for shared intermediate nodes with fermions $\left[P_{\mathrm{f}}(n)\right.$, orange points $]$ and with bosons $\left[P_{\mathrm{b}}(n)\right.$, blue triangles $]$. by a cascaded procedure with a success probability $P(n)=$ $1 / 2^{\frac{n}{2}}$.

\section{APPENDIX E: PROBABILITIES OF SUCCESS}

We finally compare the efficiency of the protocol for the various cases treated above, given the prepared state before postselection. We have already seen that, in the case of separated nodes, the success probability for both bosons and fermions is $P(n)=1 / 2^{\frac{n}{2}}$ (see green squares in Fig. 4). In the case of shared intermediate nodes, the success probabilities $P_{\mathrm{f}}(n)$ for fermions and $P_{\mathrm{b}}(n)$ for bosons decrease as a function of the particle number similarly to $P(n)$, as displayed in Fig. 4 (orange points and blue triangles, respectively). From the experimental viewpoint, one has to take into account that the requirement of Bell measurements further hinders the protocol efficiency [23,28,40-45]. Therefore, the fermionic process results in being not only qualitatively different but also more advantageous from a practical viewpoint than the other procedures that necessarily require Bell measurements.
[1] N. Gisin and R. Thew, Nat. Photon. 1, 165 (2007).

[2] D. Castelvecchi, Nature (London) 554, 289 (2018).

[3] S. Pirandola and S. L. Braunstein, Nature (London) 532, 169 (2016).

[4] P. C. Humphreys, N. Kalb, J. P. J. Morits, R. N. Schouten, R. F. L. Vermeulen, D. J. Twitchen, M. Markham, and R. Hanson, Nature (London) 558, 268 (2018).

[5] Y. Omar, Int. J. Quantum Inform. 3, 201 (2005).

[6] Y. Omar, N. Paunković, S. Bose, and V. Vedral, Phys. Rev. A 65, 062305 (2002).

[7] N. Paunković, Y. Omar, S. Bose, and V. Vedral, Phys. Rev. Lett. 88, 187903 (2002).

[8] S. Bose, A. Ekert, Y. Omar, N. Paunković, and V. Vedral, Phys. Rev. A 68, 052309 (2003).

[9] S. Bose and D. Home, Phys. Rev. Lett. 88, 050401 (2002).

[10] R. Lo Franco and G. Compagno, Sci. Rep. 6, 20603 (2016).

[11] S. Sciara, R. Lo Franco, and G. Compagno, Sci. Rep. 7, 44675 (2017).

[12] B. Bellomo, R. Lo Franco, and G. Compagno, Phys. Rev. A 96, 022319 (2017).

[13] G. Compagno, A. Castellini, and R. Lo Franco, Phil. Trans. R. Soc. A 376, 20170317 (2018).

[14] R. Lo Franco and G. Compagno, Phys. Rev. Lett. 120, 240403 (2018).

[15] F. Benatti, S. Alipour, and A. T. Rezakhani, New J. Phys. 16, 015023 (2014).

[16] D. Braun, G. Adesso, F. Benatti, R. Floreanini, U. Marzolino, M. W. Mitchell, and S. Pirandola, Rev. Mod. Phys. 90, 035006 (2018).

[17] A. Scherer, B. C. Sanders, and W. Tittel, Opt. Express 19, 3004 (2011).

[18] T.-Y. Ye, Quant. Inform. Process. 14, 1469 (2015).

[19] M. Naseri, Int. J. Theor. Phys. 55, 2428 (2016).
[20] Q.-C. Sun, Y.-L. Mao, Y.-F. Jiang, Q. Zhao, S.-J. Chen, W. Zhang, W.-J. Zhang, X. Jiang, T.-Y. Chen, L.-X. You et al., Phys. Rev. A 95, 032306 (2017).

[21] J.-W. Pan, D. Bouwmeester, H. Weinfurter, and A. Zeilinger, Phys. Rev. Lett. 80, 3891 (1998).

[22] H. de Riedmatten, I. Marcikic, J. A. W. van Houwelingen, W. Tittel, H. Zbinden, and N. Gisin, Phys. Rev. A 71, 050302(R) (2005).

[23] T. Yang, Q. Zhang, T.-Y. Chen, S. Lu, J. Yin, J.-W. Pan, Z.-Y. Wei, J.-R. Tian, and J. Zhang, Phys. Rev. Lett. 96, 110501 (2006).

[24] R. Kaltenbaek, R. Prevedel, M. Aspelmeyer, and A. Zeilinger, Phys. Rev. A 79, 040302(R) (2009).

[25] E. Megidish, A. Halevy, T. Shacham, T. Dvir, L. Dovrat, and H. S. Eisenberg, Phys. Rev. Lett. 110, 210403 (2013).

[26] Q.-C. Sun, Y.-F. Jiang, Y.-L. Mao, L.-X. You, W. Zhang, W.-J. Zhang, X. Jiang, T.-Y. Chen, H. Li, Y.-D. Huang et al., Optica 4, 1214 (2017).

[27] B. C. Jacobs, T. B. Pittman, and J. D. Franson, Phys. Rev. A 66, 052307 (2002).

[28] C. Y. Hu and J. G. Rarity, Phys. Rev. B 83, 115303 (2011).

[29] M. Zukowski, A. Zeilinger, M. A. Horne, and A. K. Ekert, Phys. Rev. Lett. 71, 4287 (1993).

[30] T. Jennewein, G. Weihs, J.-W. Pan, and A. Zeilinger, Phys. Rev. Lett. 88, 017903 (2001).

[31] C. Branciard, N. Gisin, and S. Pironio, Phys. Rev. Lett. 104, 170401 (2010).

[32] R. Horodecki, P. Horodecki, M. Horodecki, and K. Horodecki, Rev. Mod. Phys. 81, 865 (2009).

[33] S. Bose, V. Vedral, and P. L. Knight, Phys. Rev. A 57, 822 (1998).

[34] A. M. Goebel, C. Wagenknecht, Q. Zhang, Y.-A. Chen, K. Chen, J. Schmiedmayer, and J.-W. Pan, Phys. Rev. Lett. 101, 080403 (2008). 
[35] A. Khalique, W. Tittel, and B. C. Sanders, Phys. Rev. A 88, 022336 (2013).

[36] C.-Y. Lu, T. Yang, and J.-W. Pan, Phys. Rev. Lett. 103, 020501 (2009).

[37] A. N. Vamivakas, B. E. A. Saleh, A. V. Sergienko, and M. C. Teich, Phys. Rev. A 70, 043810 (2004).

[38] A. Dousse, J. Suffczyński, A. Beveratos, O. Krebs, A. Lemaître, I. Sagnes, J. Bloch, P. Voisin, and P. Senellart, Nature (London) 466, 217 (2010).

[39] A. Scherer, R. B. Howard, B. C. Sanders, and W. Tittel, Phys. Rev. A 80, 062310 (2009).

[40] S.-W. Lee and H. Jeong, Proceedings of the First International Conference on Entangled Coherent State and its Application to Quantum Information Science (Tamagawa University, Tokyo, 2012), pp. 41-46.

[41] M. Yang, W. Song, and Z.-L. Cao, Phys. Rev. A 71, 034312 (2005).

[42] R. Pakniat, M. H. Zandi, and M. K. Tavassoly, Eur. Phys. J. Plus 132, 3 (2017).

[43] W. P. Grice, Phys. Rev. A 84, 042331 (2011).

[44] L. Zhou and Y.-B. Sheng, Phys. Rev. A 92, 042314 (2015).

[45] Y.-B. Sheng, F.-G. Deng, and G. L. Long, Phys. Rev. A 82, 032318 (2010).

[46] S. Pirandola, D. Vitali, P. Tombesi, and S. Lloyd, Phys. Rev. Lett. 97, 150403 (2006).

[47] D. Press, T. D. Ladd, B. Zhang, and Y. Yamamoto, Nature (London) 456, 218 (2008).

[48] G. Fève, A. Mahé, J.-M. Berroir, T. Kontos, B. Placais, D. Glattli, A. Cavanna, B. Etienne, and Y. Jin, Science 316, 1169 (2007).
[49] E. Bocquillon, V. Freulon, J.-M. Berroir, P. Degiovanni, B. Plaçais, A. Cavanna, Y. Jin, and G. Fève, Science 339, 1054 (2013).

[50] M. Rashidi, W. Vine, T. Dienel, L. Livadaru, J. Retallick, T. Huff, K. Walus, and R. A. Wolkow, Phys. Rev. Lett. 121, 166801 (2018).

[51] J. C. Matthews, K. Poulios, J. D. Meinecke, A. Politi, A. Peruzzo, N. Ismail, K. Wörhoff, M. G. Thompson, and J. L. O’Brien, Sci. Rep. 3, 1539 (2013).

[52] L. Sansoni, F. Sciarrino, G. Vallone, P. Mataloni, A. Crespi, R. Ramponi, and R. Osellame, Phys. Rev. Lett. 108, 010502 (2012).

[53] C. Orzel, M. Walhout, U. Sterr, P. S. Julienne, and S. L. Rolston, Phys. Rev. A 59, 1926 (1999).

[54] M. Plenio and S. Virmani, Quantum Inf. Comput. 7, 1 (2007).

[55] E. Buks, R. Schuster, M. Heiblum, D. Mahalu, and V. Umansky, Nature (London) 391, 871 (1998).

[56] A. D. Armour and M. P. Blencowe, Phys. Rev. B 64, 035311 (2001).

[57] A. S. Parkins, P. Marte, P. Zoller, O. Carnal, and H. J. Kimble, Phys. Rev. A 51, 1578 (1995).

[58] L. Xiu, L. Hong-Cai, Y. Rong-Can, and H. Zhi-Ping, Chin. Phys. 16, 919 (2007)

[59] R. Pakniat, M. Tavassoly, and M. Zandi, Opt. Comm. 382, 381 (2017).

[60] C. Bäuerle, D. C. Glattli, T. Meunier, F. Portier, P. Roche, P. Roulleau, S. Takada, and X. Waintal, Rep. Prog. Phys. 81, 056503 (2018) 